\title{
Electron Energy Loss Spectroscopy of Germanium Nanowires
}

Tobias Hanrath**, JiPing Zhou ${ }^{\ddagger}$, Brian A. Korgel* ${ }^{*}$

*Dept. of Chemical Engineering, ${ }^{\ddagger}$ Texas Materials Institute, and Center for Nano- and Molecular Science and Technology

1 University Station, C 0400

University of Texas at Austin,

Austin, Texas 78712-1062

e-mail: korgel@mail.che.utexas.edu

Germanium (Ge) nanowires with diameters ranging from 6 to $50 \mathrm{~nm}$ and several $\mu \mathrm{m}$ in length were grown via a supercritical fluid-liquid-solid synthesis. ${ }^{[1]}$ A scanning electron micrograph of the raw nanowire product is shown in Figure 1a. Individual nanowires were imaged using JEOL 2010F transmission electron microscopy (TEM). Figure 1b shows a $6.5 \mathrm{~nm}$ diameter Ge nanowire with the [110] crystallographic growth direction. Parallel electron energy loss spectroscopy (PEELS) was used to study the effects of nanowire diameter on the volume plasmons, surface plasmons and core loss excitations. The position and width of the plasmon peak of nanowires of various diameters are related to quantum confinement effects, surface states, and local stress fields. In order to obtain spatially resolved information about volume and surface plasmons, PEELS linescans were acquired, as shown in Figure 2. The effects of the nanowire surface termination on the surface plasmon modes were investigated by subjecting nanowires to various wet chemical surface modifications.

The shift in plasmon peak energy can be attributed to combination of quantum confinement effects, surface states, and local stress fields due to surface reconstruction. To isolate the contribution due to quantum confinement effects the three contributing factors had to be decoupled. We intend to accomplish this by comparing the size dependent changes in the core loss spectra to size dependencies observed in plasmon loss spectra. The core loss spectra, which yield information about the changes in conduction band states, were not influenced by surface states or localized stress field. Our EELS results have further confirmed that the changes in conduction band structure are strongly related to the nano-materials size due to size quantization of the conduction electrons, as reported by Batson and Heath ${ }^{[2]}$ for the EELS study on Si nanocrystals. Similarly, Sander et al. ${ }^{[3]}$ have investigated size dependent plasmon peak energies in bismuth nanowire arrays and have found increasing plasmon peak energy with decreasing nanowire diameter.

In addition to the localized stress fields in the single crystal nanowire resulting from extensive surface reconstruction, we have also identified additional larger scale stress fields in the nanowire resulting from twisting and bending. We have studied the correlation between local stress fields and plasmon peak energies by combining highresolution TEM and nano-beam diffraction with spatially resolved PEELS. 
The Au-Ge interface present at the seed end of our nanowire structures was also investigated with PEELS mapping in an effort to understand the changes in conduction and valence band structure across this interface.

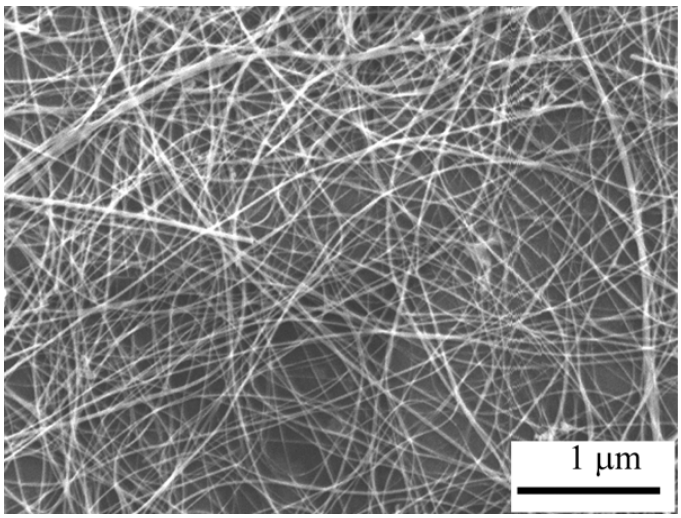

(a)

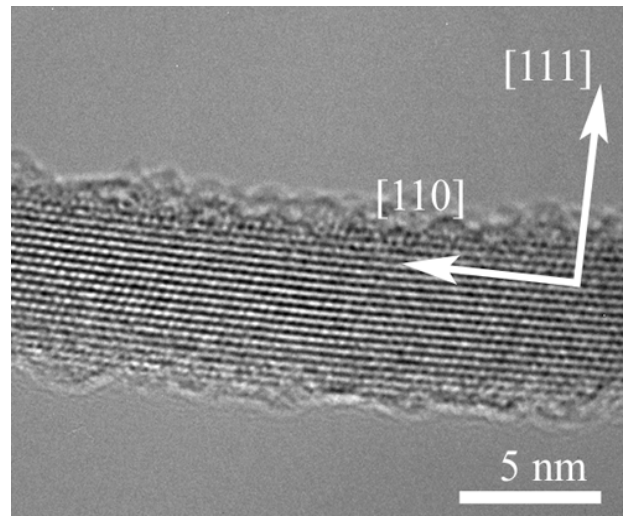

(b)

Figure 1 - (a) SEM image of bulk quantities of Ge nanowires deposited on a Si substrate. (b) high resolution TEM images showing the (111) crystal planes of the single crystal Ge nanowire with [110] crystallographic growth direction.

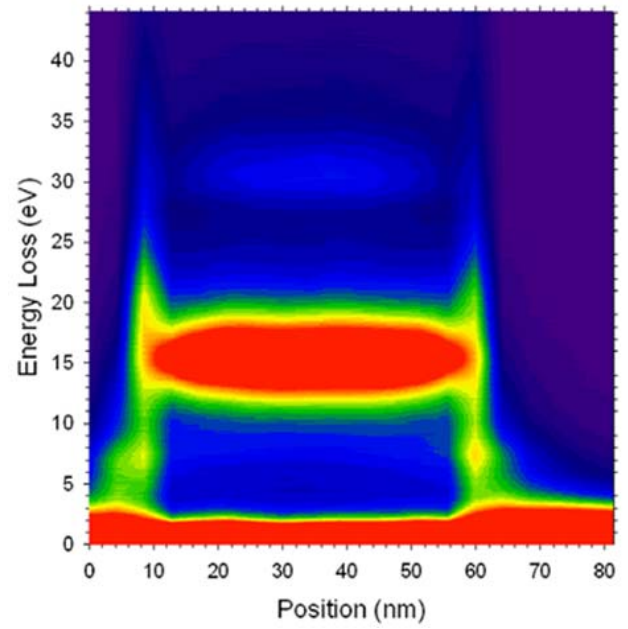

(a)

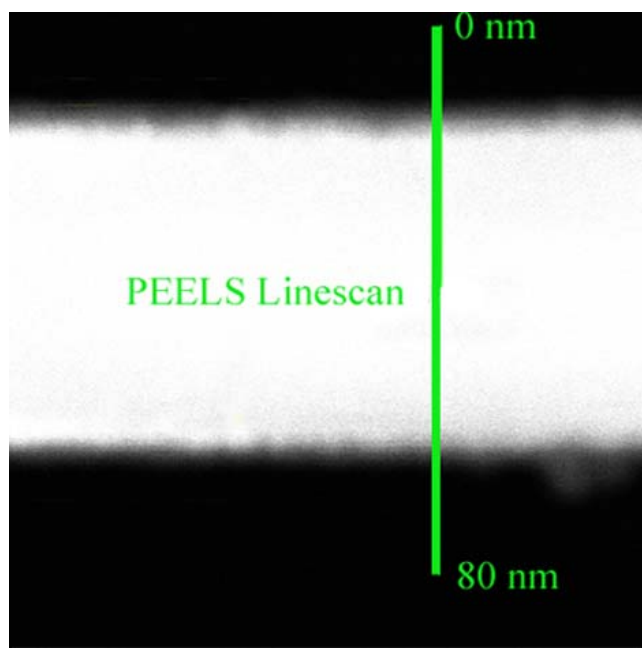

(b)

Figure 2 - (a) PEELS linescan perpendicular to nanowire axis showing surface and volume plasmons and (b) corresponding STEM image.

\section{References}

[1] T. Hanrath, B. A. Korgel, J. Am. Chem. Soc. 2001, 124, 1424.

[2] P. E. Batson, J. R. Heath, Phys. Rev. Lett. 1993, 71, 911.

[3] M. S. Sander et al., J. Appl. Phys. 2001, 89, 2733. 